\title{
Reviewers for Volume 41
}

Manju Acharya, University of Lethbridge, Lethbridge AB (Canada)

Abraham Akkerman, University of Saskatchewan, Saskatoon (Canada)

Gunnar Andersson, Stockholm University (Sweden)

Sulaiman Bah, University of Dammam (Saudi Arabia)

Alain Bélanger, INRS Centre - Urbanisation Culture Société, Québec (Canada)

Philippe Boquier, Centre de recherche en démographie et societés - Université catholique de Louvain (Belgium)

Matthew Brzozowski, York University, York ON (Canada)

Iris Chi, University of Southern California, Los Angeles (USA)

Kuate Defo Barthelemy, University of Montreal (Canada)

Frank Denton, McMaster University, Hamilton ON (Canada)

Jean-Dominique Morency, INRS Centre - Urbanisation Culture Société, Québec (Canada)

Samia El-Badry, University of Mississippi, University MS (USA)

Amir Erfani, Nipissing University, North Bay ON (Canada)

Ann Evans, Demographic \& Social Research Institute - Australian National University, Canberra (Australia)

Anna Farmer, University of Alberta, Edmonton AB (Canada)

Eric Fong, University of Toronto (Canada)

Kristyn Frank, Statistics Canada, Ottawa (Canada)

James Frideres, University of Calgary, Calgary AB (Canada)

Yuming Fu, National University of Singapore (Singapore)

Gustave Goldmann, Université d'Ottawa (Canada)

Leslie Hayduk, University of Alberta, Edmonton AB (Canada)

Charles L. Harper, Creighton University, Omaha NE (USA)

Lisa K. Jepsen, University of Northern Iowa, Cedar Falls IA (USA)

Mostafa Kamal, University of Malaya, Kuala Lumpur (Malaysia)

Etem Karakaya, Adnan Menderes University, Aydin (Turkey)

Don Kerr, King's University College at University of Western Ontario, London ON (Canada)

Mobarak H. Khan, University of Bielefeld (Germany)

Daniel Kruger, University of Michigan, Ann Arbor MI (USA)

Evelyne Lapierre-Adamcyk, Université de Montréal (Canada)

Sugie Lee, Hanyang University, Seoul (Korea)

Pierre Lefebvre, Université de Montréal (Canada)

Guillaume Lefrançois, CIM - Conseil en immobilisation \& management inc., Quebec (Canada)

Karen Leppel, Widener University, Chester PA (USA)

Jianye Liu, Lakehead University, Sudbury ON (Canada) 
M. Mazharul Islam, Sultan Qaboos University, Muscat (Sultanate of Oman)

Susan A. McDaniel, Prentice Institute for Global Population \& Economy, University of Lethbridge, Lethbridge AB (Canada)

Bruce Newbold, McMaster University, Hamilton ON (Canada)

Gerda Neyer, Stockholm University Demography Unit (SUDA), Stockholm (Sweden)

Mario Polèse, INRS Centre - Urbanisation Culture Société, Québec (Canada)

K.V. Rao, India Network Foundation, Orlando FL (USA)

Mark W. Rosenberg, Queen's University, Kingston ON (Canada)

James Saku, Frostburg State University, Frostburg MD (USA)

Grant Schellenberg, Statistics Canada, Ottawa (Canada)

Sidney Ruth Schuler, Global Health, Population and Nutrition Group, Washington DC (USA)

Andrew Sharpe, Centre for the Study of Living Standards, Ottawa (Canada)

Kara Somerville, University of Saskatchewan, Saskatoon (Canada)

Samir Soneji, Dartmouth Institute for Health Policy and Clinical Practice, Darthmouth College, Dartmouth NH (USA)

Byron Spencer, McMaster University, Hamilton ON (Canada)

David A. Swanson, University of California Riverside, Riverside CA (USA)

Ruchira Tabassum Naved, Gender, Health, Human Rights and Violence against Women Center for Equity and Health Systems, Dhaka (Bangladesh)

Zongli Tang, Auburn University at Montgomery, Montgomery AL (USA)

Frank Trovato, University of Alberta, Edmonton AB (Canada)

Lennert Veerman, University of Queensland (Australia)

Zheng Wu, University of Victoria, Vancouver (Canada) 